\title{
Skin prick testing does not reflect the presence of lgE against food allergens in adult eosinophilic esophagitis patients: a case study
}

Toral A Kamdar, Anne M Ditto, Paul J Bryce

\begin{abstract}
Skin prick testing is widely used to predict the presence of allergen-specific IgE. In eosinophilic esophagitis patients, who frequently exhibit polysensitization and broad reactivity upon skin prick testing, this is commonly used to aid avoidance recommendations in the clinical management of their disease. We present here the predictive value of skin prick testing for the presence of allergen-specific lgE, in 12 patients, determined by immunoblot against the allergen extracts using individual-matched serum. Our results demonstrate a high degree of predictive value for aeroallergens but a poor predictive value for food allergens. This suggests that skin prick testing likely identifies lgE reactivity towards aeroallergens in adult eosinophilic esophagitis but this is not true for foods. Consequently, IgE immunoblotting might be required for determining food avoidance in these patients.
\end{abstract}

\section{Background}

Eosinophilic esophagitis (EoE) is a gastrointestinal disorder that is associated with allergic disease. Studies have described personal or family histories of asthma or allergic rhinitis in adult EoE patients [1,2] and a murine model has shown that aeroallergen sensitization may be responsible for the eosinophilic response in the esophagus [3]. Adult EoE patients display a broad range of reactivity to multiple allergens that spans both aero and food allergens. In one study, $81 \%$ of EoE patients had poly-reactivity to environmental allergens on skin prick testing (SPT) [4]. In light of such broad reactivity, we queried the reliability of SPT for determining actual IgE-mediated reactions in this patient population.

\section{Case Study}

Here, we compared the presence of specific IgE (determined by Western blots) to SPT in patients with biopsy-proven EoE. Blood was drawn from $12 \mathrm{EoE}$ patients (demographics, EoE diagnostic criteria, allergic status and therapeutic treatments shown in Table 1) who were SPT positive (wheal size greater than $3 \mathrm{~mm}$ with surrounding erythema) to a minimum of one aero

\footnotetext{
* Correspondence: p-bryce@northwestern.edu

Division of Allergy-Immunology, Feinberg School of Medicine, Northwestern University, Chicago, IL 60610, USA
}

(c) 2010 Kamdar et al; licensee BioMed Central Ltd. This is an Open Access article distributed under the terms of the Creative Commons Attribution License (http://creativecommons.org/licenses/by/2.0), which permits unrestricted use, distribution, and reproduction in any medium, provided the original work is properly cited. and one food allergen. None of the patients has a history of IgE-mediated food allergy and, instead had been determined as EoE patients due to a history of dysphagia, presence of endoscopic characteristics (e.g. rings, furrows, strictures) and pathological assessment of eosinophils in esophageal biopsy tissue that were greater than 25 per high powered field (hpf). Western blotbased screening for IgE-specific recognition of proteins in 5 aero and 5 food allergen extracts was undertaken and compared to the SPT outcomes towards the same extracts. In determining the positive or negative responses by Western blot, each extract was resolved, transferred and probed with patient specific serum. The presence of IgE specific to bands within the extracts was determined using HRP-labeled anti-human IgE. While several bands were observed, the results reflect the presence or absence of any recognition.

The overall match comparison between SPT and immunoblot for all allergens was 75.6\% (Table 2). However, aeroallergens vastly outweighed food allergens in their SPT reliability. The combined reliability for aeroallergens was $89 \%$ while foods were only $56 \%$. The positive predictive value of skin testing for aeroallergens was $95 \%$, with only 3 false positives; all were to maple. Cat was responsible for most false negatives (3/12). Conversely, food allergen SPT correlated poorly with immunoblot reactivity, with false negatives common for wheat 
Table 1 Patient demographics, allergic status and treatments

\begin{tabular}{|c|c|c|c|c|c|c|c|}
\hline Patient & Age & Gender & Allergic Rhinitis & Asthma & Eos per HPF & Elimination Diet & Swallowed steroids \\
\hline$A$ & 48 & M & Yes & No & 50 & Yes & No \\
\hline B & 43 & M & Yes & Yes & $>25$ & No & No \\
\hline C & 71 & M & Yes & Yes & $>25$ & Yes & No \\
\hline$D$ & 34 & M & Yes & No & $>25$ & No & No \\
\hline E & 33 & $\mathrm{~F}$ & Yes & Yes & $>25$ & Yes & No \\
\hline F & 39 & $\mathrm{~F}$ & Yes & No & $>150$ & No & Yes \\
\hline G & 47 & $\mathrm{~F}$ & Yes & Yes & $>25$ & No & Yes \\
\hline$H$ & 42 & $\mathrm{~F}$ & Yes & Yes & $>25$ & No & No \\
\hline l & 36 & M & Yes & No & $>25$ & No & Yes \\
\hline J & 51 & $\mathrm{~F}$ & Yes & Yes & $>25$ & Yes & Yes \\
\hline K & 56 & $\mathrm{~F}$ & Yes & No & $>25$ & Yes & No \\
\hline L & 30 & $M$ & Yes & Yes & $>25$ & Yes & Yes \\
\hline$M$ & 32 & $M$ & Yes & No & $>25$ & No & Yes \\
\hline$N$ & 38 & $M$ & Yes & No & $>25$ & No & No \\
\hline 0 & 39 & $\mathrm{~F}$ & Yes & No & $>25$ & No & No \\
\hline
\end{tabular}

and whole milk (6/12 and 7/12) and false positives for peanut $(2 / 12)$. In all cases, patients displayed negative reactivity for some of the extracts, indicating the specificity of the immunoblot approach for determining positive or negative IgE binding to the specific allergen extracts.

Consequently, SPT for adult EoE patients may be a reliable method for determining IgE-associated reactivity towards aeroallergens. However, while patients demonstrated the presence of food protein-specific serum IgE, SPT appears inadequate for determining this. Alternatively, EoE patients may be refractory to food specific IgE triggering by SPT via an unknown mechanism.

\section{Conclusions}

Clinically, while elemental diet-therapy has been attempted for EoE, it is often not well-tolerated in adults due to its stringency and limitations. Instead, specific food elimination (SFE) has been explored, the foods for which are generally determined by SPT. Various studies have reported SPE as less efficacious than elemental diets [5]. This could be partially explained by poor correlation of SPT with actual IgE-specific reactivity. A report in pediatric EoE, suggested to contain both $\operatorname{IgE}$ and non-IgE reactions, concluded that a combination of SPT with atopic patch test (to identify the non-IgE reaction) might be beneficial for determining foods to eliminate for SPE [6]. Our data supports previous findings [4] that adult EoE patients are highly IgE positive but now suggests that SPT may actually fail to identify the presence of food allergen-specific IgE and that immunoblot be required to determine this. Alternatively, Immuno-CAP based determination could be useful is assessing the quantitative IgE levels, although the actual

Table 2 Results of SPT versus IgE-specific recognition by Western Blot

\begin{tabular}{lcccc}
\hline Allergen & Positive/Positive & Positive/Negative & Negative/Positive & Negative/Negative \\
\hline Cat & 7 & 0 & 3 & 2 \\
Bermuda grass & 11 & 0 & 0 & 1 \\
Blue grass & 10 & 0 & 1 & 1 \\
Dust mite & 11 & 0 & 0 & 0 \\
Ragweed & 12 & 0 & 2 & 0 \\
Maple tree & 7 & 3 & 7 & 0 \\
Wheat & 5 & 0 & 6 & 2 \\
Whole milk & 4 & 0 & 0 & 0 \\
Shrimp & 1 & 0 & 0 & 5 \\
Peanut & 5 & 2 & 3 & 1 \\
Almond $^{3}$ & 7 & 0 & 2 & 5 \\
Whole egg $^{2}$ & 1 & 4 & & \\
\hline
\end{tabular}

1. Refers to number of skin tests positive/number of western blots positive2. Only one patient had skin testing done to shrimp3. Only eleven patients had skin testing done to almond 
quantities of food allergen specific IgE are generally not thought to correlate with disease severity [7].

In conclusion, our data is the first study to determine the reliability of SPT as a detection method for determining the presence of allergen-specific IgE within adult EoE patients. Within this patient group, aeroallergen reactivity by SPT faithfully predicts the presence of specific IgE, despite such broad reactivity. Conversely, food allergens exhibit both false negatives and false positives that diminish confidence in the SPT response for concluding IgE presence. As a consequence, direct determination of food allergen-specific IgE may be beneficial in identifying the likely food triggers for clinicians considering food elimination therapy in EoE patients.

\section{Consent}

Written informed consent was obtained from all patients to allow materials to be used for research purposes and subsequent reports, under protocols approved by the Northwestern University Institutional Review Board.

\section{List of abbreviations used}

EOE: Eosinophilic esophagitis; HPF: High Power Field; IGE: Immunoglobulin E; SPE: Specific Food Elimination diet; SPT: Skin prick test.

\section{Acknowledgements}

Support: PJB was supported by funds from the Food Allergy Initiative and by NIH grant 1R01Al072570.

\section{Authors' contributions}

TK, AD, PB designed the experiments. TK, AD obtained samples. TK, PB performed the experiments. TK, PB wrote the manuscript. All authors have read and approved this work.

\section{Competing interests}

The authors declare that they have no competing interests.

Received: 6 August 2010 Accepted: 17 November 2010

Published: 17 November 2010

\section{References}

1. Arora AS, Yamazaki K: Eosinophilic esophagitis: asthma of the esophagus? Clin Gastroenterol Hepatol 2004, 2:523-530.

2. Simon D, Marti $H$, Heer $P$, Simon HU, Braathen LR, Straumann A: Eosinophilic esophagitis is frequently associated with lgE-mediated allergic airway diseases. J Allergy Clin Immunol 2005, 115:1090-1092.

3. Mishra A, Hogan SP, Brandt EB, Rothenberg ME: An etiological role for aeroallergens and eosinophils in experimental esophagitis. J Clin Invest 2001, 107:83-90.

4. Roy-Ghanta S, Larosa DF, Katzka DA: Atopic characteristics of adult patients with eosinophilic esophagitis. Clin Gastroenterol Hepatol 2008, 6:531-535.

5. Spergel JM: Eosinophilic esophagitis in adults and children: evidence for a food allergy component in many patients. Current opinion in allergy and clinical immunology 2007, 7:274-278.

6. Spergel JM, Brown-Whitehorn T, Beausoleil JL, Shuker M, Liacouras CA: Predictive values for skin prick test and atopy patch test for eosinophilic esophagitis. J Allergy Clin Immunol 2007, 119:509-511.

7. Sicherer SH, Sampson HA: Peanut allergy: emerging concepts and approaches for an apparent epidemic. J Allergy Clin Immunol 2007, 120:491-503, quiz 504-495.

\section{doi:10.1186/1476-7961-8-16}

Cite this article as: Kamdar et al.: Skin prick testing does not reflect the presence of lgE against food allergens in adult eosinophilic esophagitis patients: a case study. Clinical and Molecular Allergy 2010 8:16.

\section{Submit your next manuscript to BioMed Central and take full advantage of:}

- Convenient online submission

- Thorough peer review

- No space constraints or color figure charges

- Immediate publication on acceptance

- Inclusion in PubMed, CAS, Scopus and Google Scholar

- Research which is freely available for redistribution 